\title{
Potential Use of Shared Frameshift Mutations in 'Off-the-Shelf' Neoantigen Vaccines
}

Kerstin Thol ${ }^{1,2}$, Nicholas McGranahan ${ }^{1,2, *}$

${ }^{1}$ Cancer Genome Evolution Research Group, University College London Cancer Institute, Paul O'Gorman Building, 72 Huntley Street, London WC1E 6BT, UK

${ }^{2}$ Cancer Research UK Lung Cancer Centre of Excellence, University College London Cancer Institute, Paul O'Gorman Building, 72 Huntley Street, London WC1E 6BT, UK

*Correspondence: nicholas.mcgranahan.10@ucl.ac.uk (N. McGranahan).

\section{Abstract:}

Neoantigens derived from frameshift mutations in microsatellite unstable tumours are more commonly shared between cancer patients compared to neoantigens arising from missense mutations. A recent study by Roudko et al. evaluates the immunogenicity of shared frameshift neoantigens which could potentially be used in 'off-the-shelf' neoantigen vaccines.

\section{Main text}

Immune checkpoint inhibitors (CPIs) have revolutionised cancer treatment in a range of cancer types. By targeting antibodies that act to prevent the immune system from attacking cancer cells, these therapies can prevent tumour immune evasion, thus enabling T cells which recognise tumour cells as foreign to be activated, and ultimately facilitating an active immune response [1]. Neoantigens are cancer mutations which give rise to peptides that are recognised by the immune system as foreign (Figure 1). However, only a subset of patients benefits from these therapies, leading to a renewed interest in 'off-the-shelf' cancer vaccines.

In contrast to targeted therapies, which focus on specific actionable alterations (e.g. EGFR activating mutations) [2] which are shared in many patients' tumours, the success of immunotherapy has largely been attributed to the quantity of 'private' putative tumour neoantigens [3] which are mostly specific to each individual tumour. For instance, a study of missense mutations in colorectal cancer found that each tumour sample investigated was characterized by a distinct mutational signature and only shared up to 6 mutated cancer genes with other tumour specimens [4]. A major challenge, then, in developing targeted neoantigen directed therapies is the lack of a common target; if the neoantigen repertoire of each patient is unique, are 'off-the-shelf' approaches out of the question?

A recent study by Roudko and colleagues [5] explores shared neoantigens arising from microsatellite instable genome regions and evaluates whether these can stimulate an immune response.

Microsatellite instability describes repeated nucleotide sequences which accumulate mutations due to replication errors. Frameshift mutations derived from regions of microsatellite instability can remain in the genome due to a loss of mismatch repair function. The study by Roudko et al. focusses on microsatellite instability high (MSI-H) stomach, endometrial and colorectal cancer because of an observed increased 
microsatellite instability, increased frameshift mutation load and greater responsiveness to anti PD-1 CPIs in these cancers [6].

The authors find that frameshift mutations from microsatellite unstable regions are more likely to generate peptides shared by many patients as compared to missense mutations. In endometrial cancer, 9 shared frameshift neoantigens were found to be likely immunogenic, encode poly-epitopes, bind to a broad spectrum of MHC-I alleles and are shared by at least $20 \%$ of all MSI-H patients in the three cancer types investigated. Frameshift mutations in endometrial cancer have been less well elucidated to date and therefore became the focus of this study. The 9 neoantigens were predicted to bind to multiple frequently occurring MHC class I alleles but also to a spectrum of less frequent MHC-I alleles. Furthermore, as expected, frameshift epitopes were less similar to viral epitopes than missense mutation epitopes. Shared frameshift alleles in MSI-H tumour samples were more robustly expressed than in microsatellite stable tumour samples based on indel read counts and no evidence for epigenetic silencing of frameshift genes was observed. Tandem mass spectrometry performed on cells from the HCT116 MSI-H colorectal cancer cell line detected MSC class I binding frameshift epitopes which were predicted by shared frameshift mutations, indicating that the frameshift epitopes can be expressed and presented for immune recognition by cancer cells.

Survival analysis did not reveal significant survival benefit in any of the investigated MSI-H cancer patients from the TCGA project with a high or low frameshift load, suggesting that frameshift load is a predictive, not a prognostic biomarker. Consistently, the presence of 46 frameshift neoantigens which were shared among MSI-H stomach, colorectal or endometrial cancer samples was higher in MSI-H cancer patients responding to PD-1 blockade.

To evaluate whether these shared peptides were capable of inducing $T$ cell responses, $T$ cells from healthy donors were primed with long overlapping peptide libraries spanning each frameshift peptide in an immunogenicity assay. When combining the 9 frameshift neoepitopes a significantly increased frameshift peptide specific $T$ cell response was observed as measured by interferon- $\gamma$ production. Intracellular staining showed that responses to frameshift peptides were mainly observed in CD8+ T cells. Restimulating cells from a healthy donor which were initially expanded with pooled shared frameshift peptides showed that frameshift peptides encode multiple MHC-I restricted epitopes. High frequencies of primarily frameshift peptide specific effector CD8+ T cell responses were also detected in two MSI-H endometrial and one MSI-H colorectal cancer patient sample following stimulation with pooled shared frameshift peptides. Nevertheless, the question remains whether the shared frameshift peptides were also capable of inducing an immune response during tumour development or, as these results suggest, are widely capable of inducing an immune response in a donor environment.

Taken together, Roudko et al. show that MSI-H patients are more likely to respond to immune checkpoint blockade due to an increased neoantigen burden activating an immune response. The authors conclude that their study could be the foundation for combining anti PD-1 CPIs with 'off-the-shelf' neoantigen vaccines. 
Recently, several studies have investigated personalized but also generalized 'off-the-shelf' neoantigen vaccines. 'Off-the-shelf' vaccines have the advantage of being more affordable as well as more easily and faster to access than personalized vaccines. Leoni et al. [7] created an 'off-the-shelf' vaccine based on shared frameshift neoantigens from MSI-H patients from the colorectal, gastric and endometrial cancer TCGA cohorts. This vaccine was tested in mice and elicited a strong $T$ cell immune response, thus confirming Roudko and colleague's hypothesis that shared frameshift mutations could be used for a generalized cancer vaccine. To improve the efficacy of generalized vaccines, clonal neoantigens, i.e. neoantigens present in all cancer cells, could be investigated. A pan cancer study by Turajlic and colleagues [8] demonstrated an increased association of clonal frameshift indel mutations with response to anti PD-1 CPIs. Furthermore, a case report by Tran et al. [9] showed tumour regression following autologous cell therapy with CD8+ T cells recognizing mutant KRAS in a colorectal cancer patient. KRAS mutations are likely to be clonal and a major driver gene in several cancer types.

Personalized vaccines could potentially have greater success in highly heterogenous tumours. Keskin et al. [10] found that personalized neoantigen vaccines induced a T cell response in immune cold glioblastoma patients and that the $T$ cell responses were neoepitope specific. However, all examined patients relapsed eventually, highlighting that the immune response initiated by the neoantigen vaccine might not be strong enough or the occurrence of loss of heterozygosity at the human leukocyte antigen (HLA) locus as a potential mechanism of immune evasion at a later stage in tumour evolution [11].

In summary, the study by Roudko et al. highlights the immunogenicity of shared frameshift mutations in MSI-H tumours. This knowledge could lead the way for more effective therapy approaches and more accurate predictions of patients who will benefit from immunotherapy.

1 Topalian, S.L. et al. (2015) Immune checkpoint blockade: A common denominator approach to cancer therapy. Cancer Cell 27, 450-461

2 Cunningham, D. et al. (2004) Cetuximab Monotherapy and Cetuximab plus Irinotecan in Irinotecan-Refractory Metastatic Colorectal Cancer. N. Engl. J. Med. 351, 337-345 McGranahan, N. et al. (2016) Clonal neoantigens elicit T cell immunoreactivity and sensitivity to immune checkpoint blockade. Science. 351, 1463-1470

4 Sjöblom, T. et al. (2006) The consensus coding sequences of human breast and colorectal cancers. Science. 314, 268-274

5 Roudko, V. et al. (2020) Shared Immunogenic Poly-Epitope Frameshift Mutations in Microsatellite Unstable Tumors. Cell 183, 1634-1649.e17

6 Marabelle, A. et al. (2020) Efficacy of pembrolizumab in patients with noncolorectal high microsatellite instability/ mismatch repair-deficient cancer: Results from the phase II KEYNOTE-158 study. J. Clin. Oncol. 38, 1-10

7 Leoni, G. et al. (2020) A Genetic Vaccine Encoding Shared Cancer Neoantigens to Treat Tumors with Microsatellite Instability. Cancer Res. 80, 3972-3982

8 Turajlic, S. et al. (2017) Insertion-and-deletion-derived tumour-specific neoantigens and the immunogenic phenotype: a pan-cancer analysis. Lancet Oncol. 18, 10091021

9 Tran, E. et al. (2016) T-Cell Transfer Therapy Targeting Mutant KRAS in Cancer. N. 
Engl. J. Med. 375, 2255-2262

10 Keskin, D.B. et al. (2019) Neoantigen vaccine generates intratumoral T cell responses in phase Ib glioblastoma trial. Nature 565, 234-239

11 McGranahan, N. et al. (2017) Allele-Specific HLA Loss and Immune Escape in Lung Cancer Evolution. Cell 171, 1259-1271.e11

\section{Figure legend}

Figure 1. Differences of neoantigens derived from missense or frameshift mutations and private and shared neoantigens. (A) Frameshift mutations lead to neoantigens less similar to self than missense mutations. (B) Frameshift mutations are more commonly shared between patients than private missense mutations. 